\title{
An approach for a performance calculation of shuttle-based storage and retrieval systems with multiple-deep storage
}

\author{
Michael Eder ${ }^{1}$ iD
}

Received: 7 June 2019 / Accepted: 12 December 2019 / Published online: 20 February 2020

(C) The Author(s) 2020

\begin{abstract}
This paper presents a method to determine the performance of shuttle-based storage and retrieval systems (SBS/RS) with tier captive single-aisle shuttles and multiple-deep storage. The basis of this calculation method is a continuous-time open queueing system with limited capacity. The cycle times of lifts and shuttles, determined by a spatial value approach, can be directly used in the presented method with their time distributions. To take the multiple storage into account, a probabilitybased approach is applied. The invented approach is validated by a comparison with a discrete event simulation. A European material handling provider had given the data used in this comparison. Finally, an example is presented to outline how this calculation model can be used for designing SBS/RS which fulfil the predefined requirements. The result of this example is that with an increase of the storage depth to a certain value, the throughput increases and the cost decreases up to the same storage depth.
\end{abstract}

Keywords Automated warehouse $\cdot$ Shuttle-based storage and retrieval system $\cdot$ Multiple-deep storage $\cdot$ Analytical and numerical modeling $\cdot$ Performance analysis

\section{Introduction}

Technological developments in the global supply chain have increased the requirements for storage technology. An important part of automated warehouses that meet these requirements are autonomous vehicle storage and retrieval systems (AVS/RS). These systems work with two different types of transporters. The horizontal movement is executed by vehicles called shuttles and the vertical transport is performed by lifts. The type of AVS/RS treated in this paper is a tier-captive system. In these systems, the shuttles are confined to a tier which they cannot leave. Furthermore, the lifts only transport totes. This leads to an independence of the shuttle and lift movements. These systems are also called shuttle-based storage and retrieval systems (SBS/RS) [18].

Due to the fact that space is not an unlimited good, racks with multiple-deep storage are rising in popularity

Michael Eder

michael.e307.eder@tuwien.ac.at

1 Institute for Engineering Design and Product Development, Vienna University of Technology, Getreidemarkt 9, 1060 Wien, Austria
[15]. This requires a calculation method to describe these systems. The aim of this study is to present an analytical approach for SBS/RS with multiple-deep storage that delivers accurate results concerning the maximum throughput. Moreover, this analytical approach is devised to optimizations of SBS/RS with multiple-deep racks for preset requirements. In order to describe the existing system as close to reality as possible, the depicted approach is continuous in time and discrete in space.

The aim of this paper is to provide a decision tool that accurately and quickly evaluates the throughput of SBS/RS with multiple-deep storage. This leads to a possible use of the approach in the design process of new storage systems. The invented approach is not limited to a specific depth of storage, so the material handling provider can determine the throughput of the SBS/RS regardless of the storage depth. For reasons of space, the leading manufacturers of such SBS/RS are developing systems with up to sixfold deep storage and even deeper.

This paper is organized as follows. The first part, Section 2, is a literature review of all relevant papers on SBS/RS. The treated shuttle system and the underlying assumptions will be presented in Section 3. In Section 4, the calculation of the performance will be outlined. Especially the interarrival time, the service time of the shuttle for 
different storage depths of the rack and the open queueing model with limited capacity are being focused on. This approach takes the interactions between lifts and shuttles into account. The numerical study presented in Section 5 demonstrates the accuracy of the developed calculation model by comparison to a discrete event simulation (DES). The parameters for this numerical study are given by a European material handling provider. There will also be a comparison of different rack depths and the reachable performance of these configurations. Finally, in Section 6, the paper is summarized and an outlook on future research is given.

\section{Literature review}

In this section, the literature on tier captive SBS/RS is reviewed.

There are two ways to get accurate performance measures for SBS/RS. One way is to use a discrete event simulation (DES) to evaluate the performance of the systems. Various publications by, e.g., Ekren et al. [6, 7], Marchet et al. [24], Trummer et al. [27], Lerher et al. [16, 17, 19-21] , Kriehn et al. [13], and Ha et al. [10] treat this. Literature discussing that will not be treated in this paper in more detail because of its diverging aims to this publication.

The second way to discuss an SBS/RS is an analytical approach to evaluate the possible performance. There are three widely different types of approaches in the literature to analyze an SBS/RS.

The first type is a cycle time model of the system. These approaches are only concerned with the subsystems' lifts and shuttles. The interactions between these two subsystems are not taken into account. On these cycle time approaches, there are another two different kinds of publications. On the one hand, approaches exist which are validated through simulation (Sari et al. [25]; Lerher [15]). On the other hand, approaches that are not validated through simulation are discussed in Lerher [14], Lerher et al. [18], Lerher et al. [22], and Borovinšek et al. [1]. These publications are also not treated in more detail, because of their missing treatment of the interactions between the subsystems, lifts, and shuttles.

The second analytical approach is an approximation using an open queueing network. The interarrival and service times within this queueing network are determined by cycle time models. The open queueing network is used to take account of the interactions between lifts and shuttles. Again, there are approaches that are not validated through simulation, e.g., Heragu et al. [11], Wang et al. [29], and approaches that are validated through simulation: Marchet et al. [23], Ekren et al. [8], and Epp et al. [9]. The restriction of these approaches is that they evaluate the waiting times between lifts and shuttles. On the basis of these approaches, the time to retrieve one tote from the system can be calculated, but it is not possible to evaluate the throughput of the whole system.

The third approach is a single queueing model. There is one publication by Kartnig et al. [12] using a single Markov queue $M|G| 1$ to treat SBS/RS. This approach is validated through simulation. One point that has to be mentioned according to this publication is that the maximum waiting time has to be estimated by a simulation model. In three publications [3-5], Eder et al. developed an approach relying on a single queueing model with limited capacity $(\mathrm{M}|\mathrm{M}| 1 \mid \mathrm{K}$ and $\mathrm{M}|\mathrm{G}| 1 \mid \mathrm{K})$. Their approaches use a spacecontinuous cycle time model to evaluate the interarrival and service times of the queueing model in accordance with the reference point method of the VDI3653 [28]. This causes an estimation error because of the different speed profiles depending on the distances, which are not mentioned in this approach. In Eder's latest publication [2], the transition to a time-continuous and spatially discrete approach is effectuated.

All publications mentioned in this paper, except Lerher [15] and Eder et al. [3], only discuss single-deep racks. These two papers are using a probability-based approach to take the storage depth into account. Both approaches are limited to a storage depth of 2. Lerher [15] assumes that the rear storage locations are filled first and with a filling degree of $50 \%$, the other locations are being filled, too. Eder et al. [3] did not make such assumptions. Instead, the selection of the storage slots is random, resulting in different probabilities and times within the service time approach. Table 1 gives an overview of the publications on SBS/RS.

This literature overview shows that there are a number of publications with different queueing approaches discussing single-deep SBS/RS. The publication by Eder [2] approaches the system closest to its real behavior. The queueing system presented in this approach is timecontinuous, the evaluation of the lift interarrival times and the service times of the shuttle in this publication are spatially discrete, which also corresponds to reality. In this paper, the main idea of the approach forwarded by Eder et al. [3] will be used and advanced. The main changes were in the evaluation of the service times which were altered from space continuous to spatially discrete. This includes also the integration of the different speed profiles depending on the distances to go. The second extension is the development of an approach for higher storage depths.

\section{System description}

The system that is investigated in this paper is a tier-captive single-aisle SBS/RS for double-deep storage, as shown in 
Table 1 Literature overview

\begin{tabular}{|c|c|c|c|c|}
\hline Paper & Type of system & Type of cycle & Model & Storage depth \\
\hline Heragu et al. [11] & Tier-captive & SCC & OQN (no) & Single-deep \\
\hline Marchet et al. [23] & Tier-captive & $\mathrm{SCC}$ & OQN (yes) & Single-deep \\
\hline Ekren et al. [7] & Tier-captive & SCC/DCC & SIM & Single-deep \\
\hline Lerher et al. [21] & Tier-captive & SCC/DCC & SIM & Single-deep \\
\hline Marchet et al. [24] & Tier-captive & SCC & SIM & Single-deep \\
\hline Lerher [14] & Tier-captive & SCC/DCC & CTM (no) & Single-deep \\
\hline Sari et al. [25] & Tier-captive & SCC/DCC & CTM (yes) & Single-deep \\
\hline Kartnig et al. [12] & Tier-captive & $\mathrm{DCC}$ & SQ (yes) & Single-deep \\
\hline Trummer et al. [27] & Tier-captive & SCC/DCC & SIM & Single-deep \\
\hline Lerher et al. [18] & Tier-captive & $\mathrm{SCC} / \mathrm{DCC}$ & CTM (no) & Single-deep \\
\hline Lerher et al. [20] & Tier-captive & DCC & SIM & Single-deep \\
\hline Ekren et al. [6] & Tier-captive & DCC & SIM & Single-deep \\
\hline Wang et al. [29] & Tier-captive & SCC/DCC & OQN (no) & Single-deep \\
\hline Lerher [15] & Tier-captive & SCC/DCC & CTM (yes) & Double-deep \\
\hline Eder et al. [4] & Tier-captive & $\mathrm{SCC} / \mathrm{DCC}$ & SQLC (yes) & Single-deep \\
\hline Eder et al. [3] & Tier-captive & $\mathrm{SCC} / \mathrm{DCC}$ & SQLC (yes) & Double-deep \\
\hline Lerher et al. [22] & Tier-captive & $\mathrm{SCC} / \mathrm{DCC}$ & CTM (no) & Single-deep \\
\hline Lerher et al. [17] & Tier-captive & $\mathrm{SCC} / \mathrm{DCC}$ & SIM & Single-deep \\
\hline Lerher et al. [19] & Tier-captive & $\mathrm{SCC} / \mathrm{DCC}$ & SIM & Single-deep \\
\hline Borovinšek et al. [1] & Tier-captive & $\mathrm{SCC} / \mathrm{DCC}$ & CTM (no) & Single-deep \\
\hline Ekren et al. [8] & Tier-captive & $\mathrm{SCC} / \mathrm{DCC}$ & OQN (yes) & Single-deep \\
\hline Lerher [16] & Tier-captive & DCC & SIM & Single-deep \\
\hline Epp et al. [9] & Tier-captive & SCC/DCC & OQN(yes) & Single-deep \\
\hline Eder et al. [5] & Tier-captive & SCC/DCC & SQLC (yes) & Single-deep \\
\hline Kriehn et al. [13] & Tier-captive & SCC/DCC & SIM & Single-deep \\
\hline Ha et al. [10] & Tier-captive & SCC & SIM & Single-deep \\
\hline Eder [2] & Tier-captive & SCC/DCC & SQLC (yes) & Single-deep \\
\hline
\end{tabular}

$S C C$ single command cycle, $D C C$ dual command cycle, $C T M$ cycle time model, $O Q N$ open queueing network, $S Q$ single queue, $S Q L C$ single queue with limited capacity, SIM simulation model

(yes)/(no): analytical model results are validated through simulation

Fig. 1. A lift that rides in vertical direction and transports the totes from the $\mathrm{I} / \mathrm{O}$ point to the tier and back is positioned in front of each rack. The I/O points are located on the first tier in front of the lifts. Buffers are placed between lifts and shuttles in each tier. Each shuttle is assigned to one tier in one aisle, meaning that per rack, the number of vehicles equals that of the tiers. Moreover, these vehicles can handle one tote at a time. The racks are multiple-deep, double-sided and each storage location can hold one tote.

The main assumptions and notations are listed below. The assumptions made here are similar to an SBS/RS produced by a European material handling provider. These assumptions have also been made in other publications of Eder et al. [2-5].
- Both lifts serve the transactions in single command cycles under an FCFS rule, one lift for the input-cycle and one for the output-cycle.

- The shuttles serve the transactions in single and double cycles under an FCFS rule.

- The dwell point of the input lift is the I/O.

- The dwell point of the output lift is the point of service completion.

- The dwell point of the shuttle is the point of service completion.

- The lifts and shuttles accelerate/decelerate in a constant manner. If not, an acceleration/deceleration rate has to be calculated that displays the same behavior as the real, variable acceleration/deceleration. 

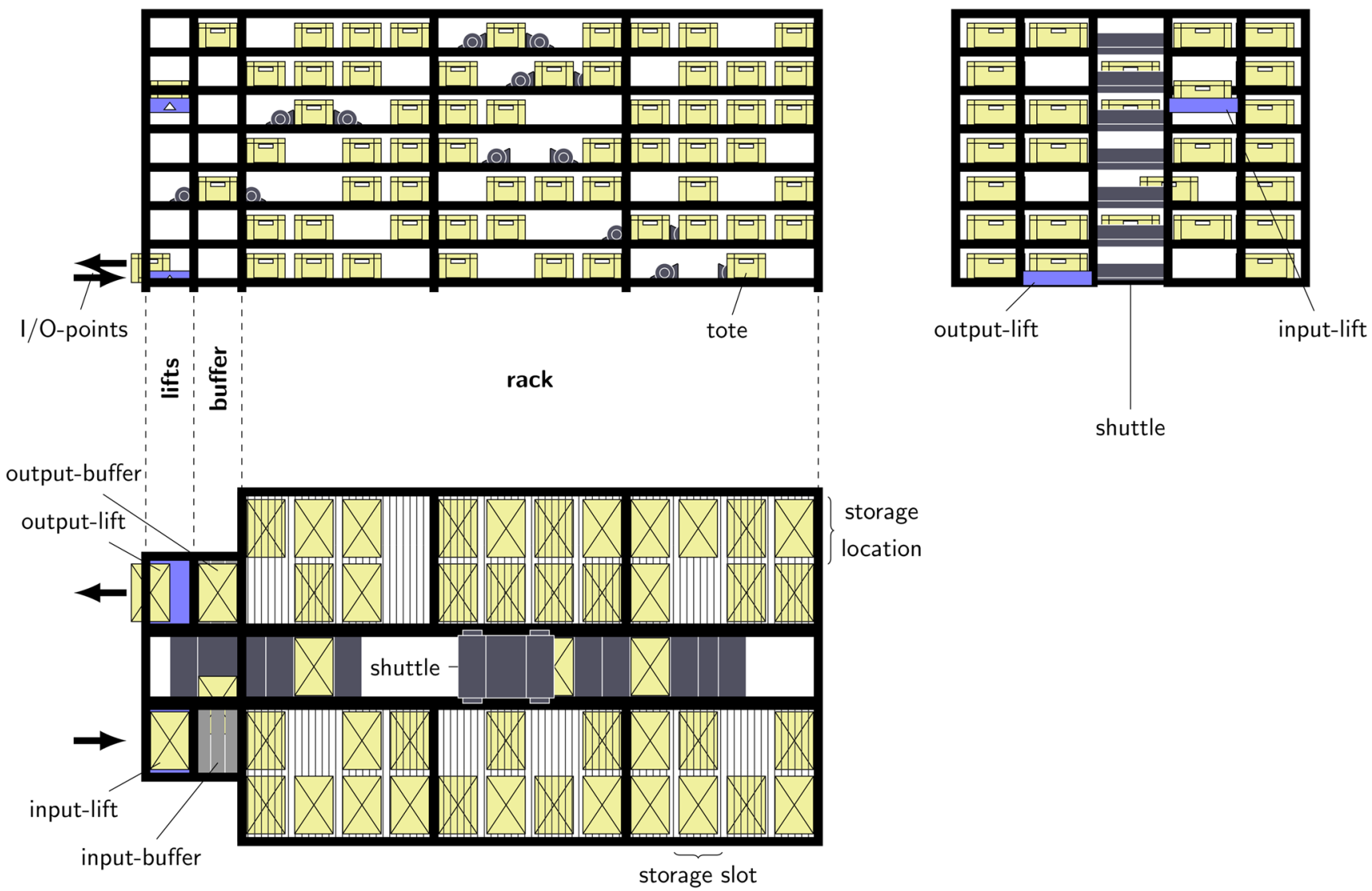

Fig. 1 Shuttle system

- There are different transfer times from and to the shuttle depending on the depth of the rack.

- There is no difference in time between the transfer of totes to and from the lifts. If there is a difference in the real system, there is no influence on the calculation, because only the sum of times for loading and unloading is used.

- There are always totes waiting on the $\mathrm{I} / \mathrm{O}$ point to be stored. This assumption is necessary to achieve the maximum throughput. Otherwise, the input lift would have to wait for incoming totes which would affect the possible performance of the SBS/RS under consideration.

- The totes are stored in an evenly distributed manner throughout the whole storage rack.

- The order in which totes should be restored is evenly distributed among all stored totes.

- The tote that has to be restored is restored to the next free storage location.

- The filling degree is limited to a certain value to enable a relocation cycle.

\section{Analytical approach}

To determine the performance of an SBS/RS system, one aisle shall be modeled. According to Epp et al. [9], Marchet et at. [23], and Heragu et al. [11], the performance of the system can be evaluated by modeling just one aisle because the storage and retrieval transactions are evenly distributed among all aisles and tiers.

The analytical approach for multiple-deep storage presented in this paper is based on the analytical approach as found in Eder [2]. This approach uses an open queueing model with limited capacity $(\mathrm{M}|\mathrm{G}| 1 \mid \mathrm{K})$. The process of this queueing model is shown in Fig. 2. This model analyzes three main parts to determine the throughput, the interarrival time to one single tier, the service time of the shuttles, and the open queueing model $\mathrm{M}|\mathrm{G}| 1 \mid \mathrm{K}$. To adopt this approach, several changes have to be made and new relations have to be established.

The procedure of this approach is (see Fig. 3):

- Determination of the interarrival time of the totes in each storage level by the lifts. 


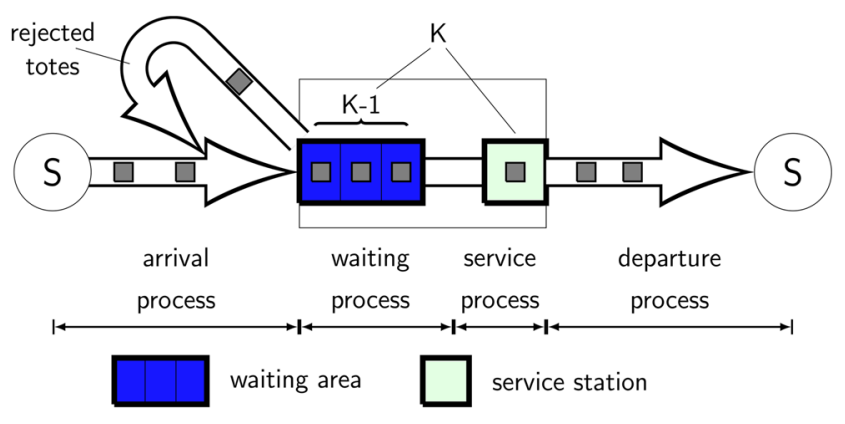

Fig. 2 Open queueing model with limited capacity [2]

- Determination of the service time within one storage level of the shuttles.

- Determination of the time for the ride within one storage level of the shuttles.

- Determination of the mean time needed to transfer the tote to and from the shuttle.

- Determination of the probability that a relocation of a tote is needed.

- Determination of the time required to ride in the relocation cycle.

- Determination of the mean time needed to transfer the tote to and from the shuttle in the relocation cycle.

- Determination of the mean time required for the relocation cycle.

- Determination of the throughput by using the open queueing model $\mathrm{M}|\mathrm{G}| 1 \mid \mathrm{K}$.

The abbreviations in this article are listed in Table 2. The notations used in this analytical approach are listed in Table 3.

\subsection{Interarrival time}

The first part in the calculation of the throughput of one tier is the interarrival time determined by the lifts. Therefore, the time for the ride and the times for transferring the tote to and from the lift are needed [2].

$t_{\text {lift }}=2 \cdot t_{R_{L}}+t_{t_{L}}$

The mean time needed for the ride is [2]:

$t_{R_{L}}=\frac{1}{n_{\text {tier }}} \sum_{k=1}^{n_{\text {tier }}} \mathbf{t}\left(\left|l_{I / O}+(k-1) \cdot \Delta y\right|\right)$

Taking into account that the lifts do not reach their maximum speed over short distances, the function $\mathbf{t}(l)$ has to be split into two sections. A part for distances below $l<\frac{v^{2}}{a}$ :

$\mathbf{t}(l)=2 \sqrt{\frac{l}{a_{\text {lift }}}}$
And another part for longer distances:

$\mathbf{t}(l)=\frac{l}{v_{\text {lift }}}+\frac{v_{\text {lift }}}{a_{\text {lift }}}$

Equation 1 is the lift's cycle time for one single command lift cycle. The interarrival time is this cycle time multiplied by the number of tiers [2].

$t_{A}=t_{\text {lift }} \cdot n_{\text {tier }}$

This equation regards the fact that one lift serves all tiers of an aisle.

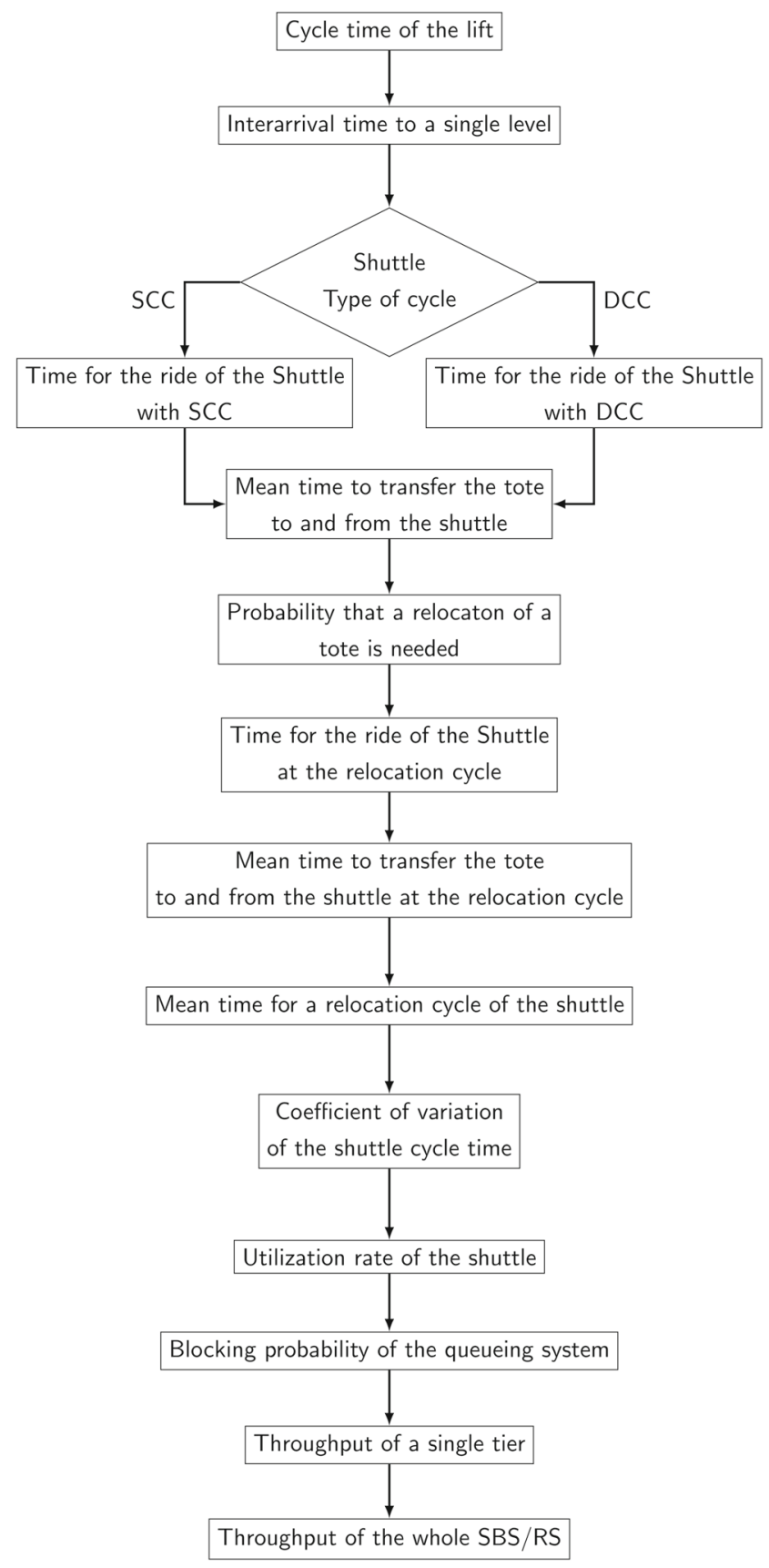

Fig. 3 Flowchart of the procedure of this approach 
Table 2 Abbreviations of the tier-captive single-aisle AVS/RS

\begin{tabular}{ll}
\hline AVS/RS & Automated vehicle storage and retrieval system \\
CTM & Cycle time model \\
DCC & Dual command cycle \\
DES & Discrete event simulation \\
FCFS & First come first served \\
OQN & Open queueing network \\
SBS/RS & Shuttle-based storage and retrieval system \\
SCC & Single command cycle \\
SIM & Simulation model \\
SQ & Single queue queueing model \\
SQLC & Single queue queueing model \\
& with limited capacity \\
\hline
\end{tabular}

\subsection{Service time}

The second part needed to determine the throughput is the service time of the shuttles. This part contains the same arguments as the determination of the cycle times of the lifts. A distinction has to be made between the times for a ride from $\mathrm{A}$ to $\mathrm{B}$ and the times required for transferring the totes to and from the shuttle.

For single command cycle and single-deep storage, the following equation is made [2].

$t_{\text {shuttle }_{\mathrm{SC}}}=2 \cdot t_{R_{S_{-} \mathrm{SC}}}+t_{t_{S}}$

The mean time for the ride results from [2]:

$t_{R_{S_{-} \mathrm{SC}}}=\frac{1}{n_{\text {slot }}} \sum_{k=1}^{n_{\text {slot }}} \mathbf{t}(k \cdot \Delta x)$

Equations 3 and 4 have to be used in order to consider the different equations depending on the distance.

The service time of the shuttles is as follows [2]:

$t_{S_{\mathrm{SC}}}=2 \cdot t_{\text {shuttle }_{\mathrm{SC}}}$

The multiplier 2 has to be used in this equation because a dual handling cycle is the reference.

For the dual command cycle and single-deep storage, the following equation is made [2]:

$t_{\text {shuttle }_{\mathrm{DC}}}=2 \cdot t_{R_{S_{-} \mathrm{SC}}}+t_{R_{S_{-} \mathrm{DC}}}+2 \cdot t_{t_{S}}$

The first term is the same as for single cycle. The second term represents the ride between the slot where the stored tote is located and the tote that shall be retrieved is located.
The mean time for this follows from [2]:

$t_{R_{S \_\mathrm{DC}}}=\frac{1}{n_{\text {slot }}^{2}} \sum_{k=1}^{n_{\text {slot }}} \sum_{l=1}^{n_{\text {slot }}} \mathbf{t}(|(k \cdot \Delta x-l \cdot \Delta x)|)$

The service time of the shuttles with double cycle is as follows [2]:

$t_{S_{\mathrm{DC}}}=t_{\text {shuttle }_{\mathrm{DC}}}$

To determine the throughput of a multiple-deep rack, the service times for single-deep racks have to be extended by the probability of relocation $\left(w_{\text {rel }}\right)$ and the time that is needed for this relocation $\left(t_{\mathrm{rel}}\right)$. For shuttles that operate single-command cycles, the equation for the service time is as follows:

$t_{S_{\mathrm{SC}}}=t_{\text {shuttle }_{\mathrm{SC}}}=4 \cdot t_{R_{S_{-} \mathrm{SC}}}+t_{t_{S}}+w_{\mathrm{rel}} \cdot t_{\mathrm{rel}}$

For dual command cycle, it is:

$t_{S_{\mathrm{DC}}}=t_{\text {shuttle }_{\mathrm{DC}}}=2 \cdot t_{R_{S \_\mathrm{SC}}}+t_{R_{S-\mathrm{DC}}}+t_{t_{S}}+w_{\mathrm{rel}} \cdot t_{\mathrm{rel}}$

Another aspect that has to be changed is the time for the transfer to and from the shuttle $\left(t_{t_{S}}\right)$. This is because the time for transferring the tote to and from the shuttle depends on which storage location the tote is located in the rack slot. This is shown in Fig. 4 for a double-deep rack.

To calculate these terms, the following points have to be determined first:

- Adaptations of the shuttles' cycle times without any relocation cycle

- Mean time to transfer the tote to and from the shuttle $\left(t_{t_{S}}\right)$ (see Section 4.2.1).
Fig. 4 A tier with double-deep racks

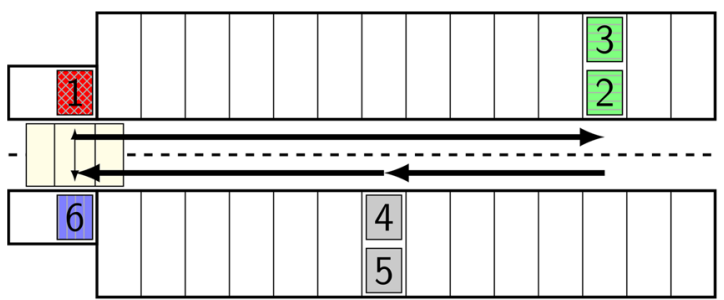

transfer to the shuttle out of the buffer transfer from the shuttle to the rack transfer to the shuttle out of the rack transfer from the shuttle to the buffer $\downarrow \uparrow$ shuttle 
Table 3 Notation of the tier-capative single-aisle AVS/RS

\begin{tabular}{|c|c|}
\hline$\Delta x$ & Distance between two storage slots \\
\hline$\Delta y$ & Distance between two tiers \\
\hline$\vartheta$ & Throughput of a single aisle \\
\hline$\vartheta_{\text {tier }}$ & Throughput of a single tier \\
\hline$\rho$ & Utilization rate of the shuttle \\
\hline$a_{\text {lift }}$ & Lift acceleration/deceleration rate \\
\hline$a_{\text {shuttle }}$ & Shuttle acceleration/deceleration rate \\
\hline$f$ & Filling degree of the storage rack \\
\hline$K$ & Capacity of the queueing system \\
\hline$l_{I / O}$ & $\begin{array}{l}\text { Vertical distance between } \\
\text { the first tier and the I/O point }\end{array}$ \\
\hline$n_{b u f}$ & $\begin{array}{l}\text { Number of buffers on each side } \\
\text { of the aisle per tier }\end{array}$ \\
\hline$n_{\text {slot }}$ & $\begin{array}{l}\text { Number of storage slots on each side } \\
\text { of the aisle per tier }\end{array}$ \\
\hline$n_{\text {tier }}$ & Number of tiers \\
\hline$p_{0}$ & $\begin{array}{l}\text { Probability of emptiness of } \\
\text { a queueing system }\end{array}$ \\
\hline$p_{k}$ & Blocking probability of a queueing system \\
\hline$s d$ & Storage depth of the rack \\
\hline$t_{A}$ & Interarrival time to a tier \\
\hline$t_{t_{L}}$ & $\begin{array}{l}\text { Time required to transfer a tote } \\
\text { to and from the lift }\end{array}$ \\
\hline$t_{t_{S}}$ & $\begin{array}{l}\text { Time required to transfer a tote } \\
\text { to and from the shuttle }\end{array}$ \\
\hline$t_{t_{S s e l}}$ & $\begin{array}{l}\text { Time required to transfer a tote } \\
\text { to and from the shuttle } \\
\text { in the relocation cycle }\end{array}$ \\
\hline$t_{l i f t}$ & $\begin{array}{l}\text { Cycle time of a lift for } \\
\text { a single command cycle }\end{array}$ \\
\hline$t_{\text {rel }}$ & Estimated time for the relocation cycle \\
\hline$t_{R_{L}}$ & $\begin{array}{l}\text { Time required by a lift to travel } \\
\text { in single command cycle }\end{array}$ \\
\hline$t_{R_{S_{-} S C}}$ & $\begin{array}{l}\text { Time required by a shuttle to travel } \\
\text { in single command cycle }\end{array}$ \\
\hline$t_{R_{S_{-} D C}}$ & $\begin{array}{l}\text { Additional time of a shuttle to travel } \\
\text { in dual command cycle }\end{array}$ \\
\hline$t_{R_{\text {_rel }}}$ & $\begin{array}{l}\text { Time required by the shuttle to travel } \\
\text { in the relocation cycle }\end{array}$ \\
\hline$t_{S}$ & Service time of a shuttle \\
\hline$t_{S_{S C}}$ & $\begin{array}{l}\text { Service time of a shuttle } \\
\text { in single command cycle }\end{array}$ \\
\hline$t_{S_{D C}}$ & $\begin{array}{l}\text { Service time of a shuttle } \\
\text { in dual command cycle }\end{array}$ \\
\hline$t_{\text {shuttle }}$ SC & $\begin{array}{l}\text { Cycle time of a shuttle for } \\
\text { a single command cycle }\end{array}$ \\
\hline$t_{\text {shuttle }_{D C}}$ & $\begin{array}{l}\text { Cycle time of a shuttle for } \\
\text { a dual command cycle }\end{array}$ \\
\hline$t_{t n}$ & $\begin{array}{l}\text { Time to transfer a tote from and to } \\
\text { the shuttle to/out of the } n \text {-th slot next }\end{array}$ \\
\hline
\end{tabular}

Table 3 (continued)

\begin{tabular}{ll}
\hline$\Delta x$ & Distance between two storage slots \\
\hline$t_{t_{b}}$ & to the aisle \\
& $\begin{array}{l}\text { Time to transfer a tote from and to } \\
\text { the shuttle to/out of the buffer }\end{array}$ \\
$s$ & Coefficient of variation of the cycle times \\
$v_{l i f t}$ & Lift velocity \\
$v_{\text {shuttle }}$ & Shuttle velocity \\
$w_{\text {rel }}$ & Probability of relocation cycle \\
\hline
\end{tabular}

- Adaptations concerning the relocation cycle

- Probability that a relocation cycle is needed $\left(w_{\text {rel }}\right)$ (see Section 4.2.2).

- Mean time needed for the ride for relocation $\left(t_{R_{\text {_rel }}}\right)$ (see Section 4.2.3).

- Mean time necessary to transfer the tote to and from the shuttle in the relocation cycle $\left(t_{t_{\text {S_rel }}}\right)$ (see Section 4.2.4).

- Mean time for the relocation cycle $\left(t_{\text {rel }}\right)$ (see Section 4.2.5).

\subsubsection{Mean time needed to transfer the tote to and from the shuttle}

For double-deep racks, the mean time can be calculated as follows:

$t_{t_{S}}=t_{t b}+\frac{1}{2} f \cdot t_{t 1}+\left[\frac{1}{2} f+(1-f)\right] t_{t 2}$

The first term $\left(t_{t b}\right)$ in this equation is the time needed to transfer the tote to and from the shuttle to/from the buffer (see Fig. 4 tote number 1 and tote number 6). The second term $\left(\frac{1}{2} f \cdot t_{t 1}\right)$ stands for the mean transfer time to and from the shuttle to/from the storage location next to the aisle (see Fig. 4 tote number 2 and tote number 4 ). The factor $(f)$ is the filling degree of the storage system as well as the probability that a storage location is occupied. The factor $\frac{1}{2}$ in this term represents the fact that, in double-deep storage, the totes not located next to the aisle are ordered with the same probability as the totes located next to the aisle. The third term $\left(\left[\frac{1}{2} f+(1-f)\right] t_{t 2}\right)$ describes the time needed for the transfer to and from the shuttle out of and to the storage location which is not situated next to the aisle (see Fig. 4 tote number 3 and tote number 5). This term covers two possibilities, the first being that in the retrieval process, the storage location next to the aisle is occupied and the tote in this storage location is not ordered $\left(\frac{1}{2} f\right)$. The second describes the transfer to and from a storage location which is not next to the aisle. The probability of a transfer to and from this storage location is $(1-f)$. These two probabilities 
are multiplied by the time needed to transfer a tote from a storage location not situated next to the aisle.

For multiple-deep storage with the storage depth $(s d)$, the mean time for the transfer is as follows:

$t_{t_{S}}=t_{t b}+\sum_{n=1}^{s d} \sum_{i=0}^{n-1} \frac{1}{s d-i}\left(\begin{array}{c}s d-1 \\ i\end{array}\right) f^{s d-1-i}(1-f)^{i} t_{t n}$

\subsubsection{Probability of relocation}

The probability of relocation evolves around the fact that the ordered tote is not directly retrievable. In Fig. 4, for example, this means that tote 5 is ordered but the forward storage location is occupied with tote 4 which consequently has to be restored first. The equation describing this probability for double-deep storage is following:

$w_{\text {rel }}=\frac{1}{2} f^{2}$

The factor $f^{2}$ in this equation describes the fact that both storage locations of one slot are occupied. The factor $\frac{1}{2}$ represents the fact that a relocation is necessary if the tote located further from the aisle is ordered.

For greater storage depths, the probability of a relocation cycle is as follows:

$w_{\text {rel }}=\sum_{n=0}^{s d-2} \sum_{i=1}^{s d-1-n} \frac{i}{s d-n}\left(\begin{array}{c}s d \\ n\end{array}\right) f^{s d-n} \cdot(1-f)^{n}$

\subsubsection{Mean time necessary for the ride in the relocation cycle}

The distances in the relocation cycle depend on the free storage locations next to a storage slot in which the ordered tote is placed. The mean distance describes the average length that must be covered during the relocation cycle. This parameter does not describe the distance for a single relocation cycle, but through a few relocation cycles, this parameter provides an accurate approximation to reality. In Fig. 5, the possible relocation locations are shown. This cycle sequence can also be described in the following equation that is also valid for greater storage depths.

$t_{R_{\text {rel }}}=\sum_{n=1}^{\frac{n_{\text {slot }}}{2}} f^{s d} \cdot\left[\left(f^{s d}\right)^{4}\right]^{n-1} \cdot\left[1-\left(f^{s d}\right)^{4}\right] \cdot \mathbf{t}(n \cdot \Delta x)$

The upper range of the sum is set to half the length of the aisles; this is the longest possible distance if the slot containing the wanted tote is located in the middle of the aisle. In Fig. 6, the probability of riding $n \cdot \Delta x$ is visualized,
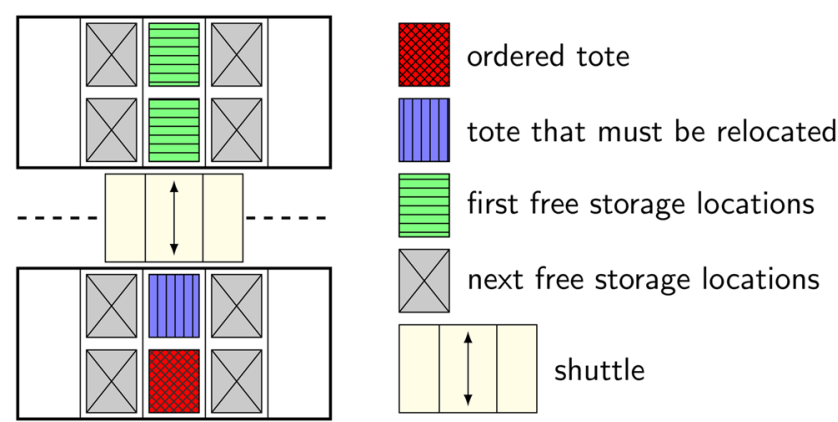

Fig. 5 Distances in the relocation cycle

with the assumed conditions of a filling degree of $90 \%$ and fivefold-deep storage. It can be seen that the probability decreases very fast so the upper range of Eq. 18 can be set to half the length of an aisle. The first term $f^{s d}$ in this equation describes the probability that the slot on the opposite side of the slot in question is fully occupied. The next term $\left[\left(f^{s d}\right)^{4}\right]^{n-1}$ represents the state that the slots beside the slot beside are fully occupied. The exponent in this term refers to the number of occupied slots on each side of the considered slot. The term $\left[1-\left(f^{s d}\right)^{4}\right]$ describes the status that at least one storage location of the four slots beside is free to store a tote. The time function $\mathbf{t}(n \cdot \Delta x)$ depending on the distance are those in Eqs. 3 and 4.

\subsubsection{Mean time needed to transfer the tote to and from the shuttle in the relocation cycle}

The mean time needed to transfer the tote from and to the shuttle in the relocation cycle is similar to the mean time

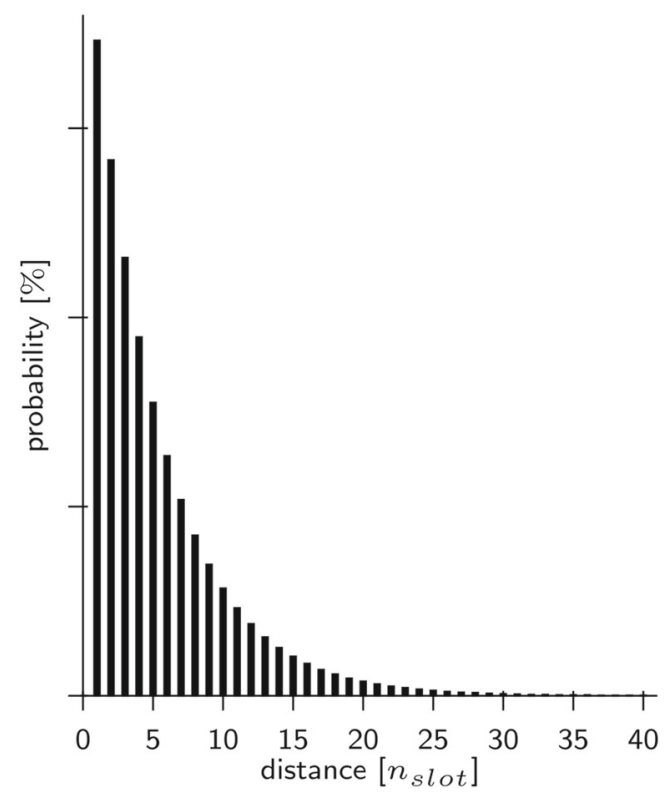

Fig. 6 Probabilities of the distance to ride in the relocation cycle with a filling degree of $90 \%$ and fivefold-deep storage 
necessary to transfer the tote from and to the shuttle, Eq. 14. The difference being the absence of a transfer from and to the buffer. The equation for double-deep storage racks is as follows:

$t_{t_{\text {S rel }}}=\frac{1}{2} f \cdot t_{t 1}+\left[\frac{1}{2} f+(1-f)\right] t_{t 2}$

For greater storage depths, the following equation was developed:

$t_{t_{S_{\text {_rel }}}}=\sum_{n=1}^{s d} \sum_{i=0}^{n-1} \frac{1}{s d-i}\left(\begin{array}{c}s d-1 \\ i\end{array}\right) f^{s d-1-i}(1-f)^{i} t_{t n}$

\subsubsection{Mean time needed in the relocation cycle}

The mean time needed in the relocation cycle is the summation of the mean time necessary for the ride $t_{R_{-} \text {rel }}$ (4.2.3) and the mean time needed to transfer the tote to and from the shuttle during the relocation cycle $t_{t_{S_{\text {_rel }}}}(4.2 .4)$.

$t_{\text {rel }}=t_{R_{-} \text {rel }}+t_{t_{\text {S_rel }}}$

\subsection{Open queueing model M | G | 1 | K}

To evaluate the influence of the buffers and the influence of the interaction between lifts and shuttles, a time-continuous open queueing model with limited capacity is used. With the help of this model, the throughput $\vartheta_{\text {tier }}$ of one single tier can be calculated [2]:

$\vartheta_{\text {tier }}=\frac{1}{t_{A}} \cdot\left(1-p_{k}\right)$

$\vartheta_{\text {tier }}=\frac{1}{t_{S}} \cdot\left(1-p_{0}\right)$

There are two methods to determine the throughput. The first method is based on the interarrival time and the blocking probability (22). The second is the use of the service time and the probability of emptiness of the queuing system (23). The term blocking probability means that the entire system is filled and lacking space for more totes to enter it. Applied to a shuttle system, this means that the lift must wait for an empty space in the input buffer. The probability of emptiness means that the server must wait because there is no tote in the queuing system. In an SBS/RS, this means that the shuttle has to wait for a tote.

The throughput of an aisle is equal to the throughput of one tier multiplied by the number of tiers [2]:

$\vartheta=\vartheta_{\text {tier }} \cdot n_{\text {tier }}$
The blocking probability of a queueing system is calculated as follows [26]:

$p_{k}=\frac{\rho^{\frac{\sqrt{\rho} \cdot s^{2}-\sqrt{\rho}+2 K}{2+\sqrt{\rho} \cdot s^{2}-\sqrt{\rho}}} \cdot(\rho-1)}{\rho^{2 \cdot \frac{\sqrt{\rho} \cdot s^{2}-\sqrt{\rho}+K+1}{2+\sqrt{\rho} \cdot s^{2}-\sqrt{\rho}}}-1}$

Despite the relatively complex appearance of this equation, it contains only three arguments. The main argument is the utilization of the service station (=shuttle). This rate is provided by the subsequent equation, which comprises the interarrival time set by the lift and the service time of the shuttle [2]:

$\rho=\frac{t_{S}}{t_{A}}$

$K$ is the capacity of the queueing system. It is the sum of the number of buffer spaces and the capacity of the shuttles, which is always one tote in the discussed SBS/RS [2].

$K=n_{\text {buf }}+1$

The third argument is $s$, the coefficient of variation of the service process. This coefficient can be calculated similarly for single-deep racks [2]. The coefficient of variation for shuttles performing single handling cycles can be calculated as follows [2]:

$s=\frac{\sqrt{\frac{\left(2 \cdot \frac{n_{\text {slot }} \Delta x}{v_{\text {shuttle }}}\right)^{2}}{12}}}{t_{S}}$

And the formula for double handling cycles is [2]:

$s=\frac{\sqrt{\frac{\left(2 \cdot \frac{n_{\text {slot }} \cdot \Delta x}{v_{\text {shuttle }}}\right)^{2}}{18}}}{t_{S}}$

These simple equations can be used because the coefficient of variation for single-deep storage is always higher than for multiple-deep storage, and thus the resulting error is less than $10 \%$. This causes a difference on the throughput that is less than $2 \%$ and the resulting error can be neglected. The service times for double cycles with singledeep storage are represented by a right-skewed triangular distribution (see Fig. 7). The service times for double cycles with fivefold-deep storage are a superposition of the service time of single-deep storage with the time needed for the relocation and the different times needed to transfer the tote to and from the shuttle depending on the storage depth (see Fig. 8 for a filling degree of 90\%). The difference between the coefficients of variation of the service times is very small, $s_{\text {single deep }}=0,28$ for single-deep storage and $s_{\text {fivefold deep }}=0,27$ for fivefold depth and a filling degree of $90 \%$. 


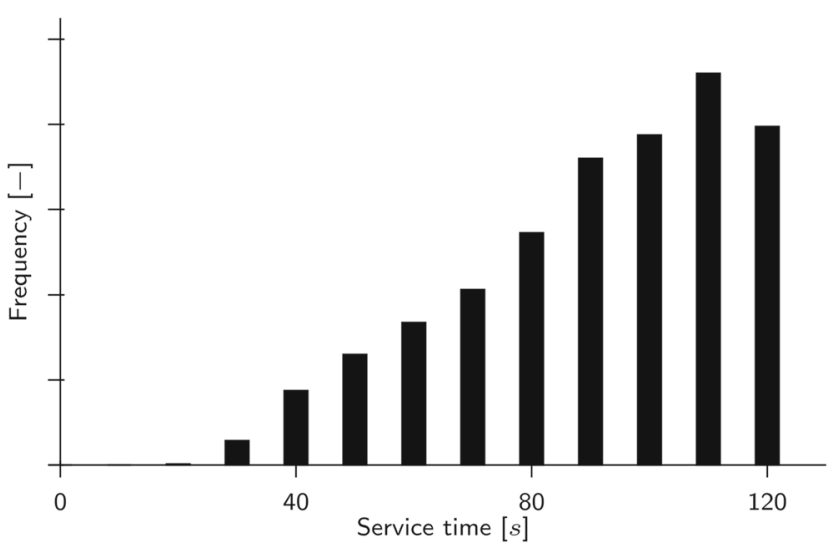

Fig. 7 Service time distribution for shuttles operating dual command cycles and single-deep storage with a filling degree of $90 \%$

The probability of emptiness of the queueing system contains the same arguments as the blocking probability and can be calculated as follows [2]:

$$
p_{0}=\frac{\rho-1}{\rho^{2 \cdot \frac{\sqrt{\rho} \cdot s^{2}-\sqrt{\rho}+K+1}{2+\sqrt{\rho} \cdot s^{2}-\sqrt{\rho}}}-1}
$$

\section{Numerical study}

The approach of the whole aisle by treating only one tier has to be validated, wherefore the analytical model is validated by comparing it to a DES. This comparison is shown in Section 5.1. Section 5.2 will outline how to use the model to design SBS/RS meeting the given requirements. An example will illustrate the specific impact of the rack's storage depth on the throughput of the system.

\subsection{Numerical evaluation of the approximation quality}

In the design process of an SBS/RS, it is important to know the performance of an aisle to design the other parts of a storage system, e.g., the following conveyor. The knowledge of the influence of the different design parameters helps to find an economically and ecologically ideal design of an SBS/RS.

To present a variety of different settings, certain parameter configurations, shown in Table 4, are selected. The system under discussion has up to 50 tiers and up to 200 slots on each side of the aisle. The storage depth of the rack is up to fivefold-deep. The parameters in Table 4 were specified by a European material handing provider of SBS/RS

For the validation, the results of the analytical model were compared to the ensemble of 30 independent replications of the simulation model. In each replication, 10,000 totes were stored and retrieved. The simulation runs were performed by the DES software SIMIO (version 10). The ordered totes are selected randomly. One storage and retrieval transaction consists of the following steps.

1. Tote is waiting for the lift.

2. Lift travels to the $\mathrm{I} / \mathrm{O}$ point.

3. Tote is transferred from the $\mathrm{I} / \mathrm{O}$ point to the lift.

4. Lift travels to the destination tier.

5. Tote is transferred from the lift to the storage buffer.

6. Tote is waiting for the shuttle.

7. Shuttle travels to the input buffer.

8. Tote is transferred from the buffer to the shuttle.

9. Shuttle travels to the storage position.

10. Tote is transferred from the shuttle to the storage rack.
Fig. 8 Service time distribution for shuttles operating dual command cycles and fivefold-deep storage with a filling degree of $90 \%$

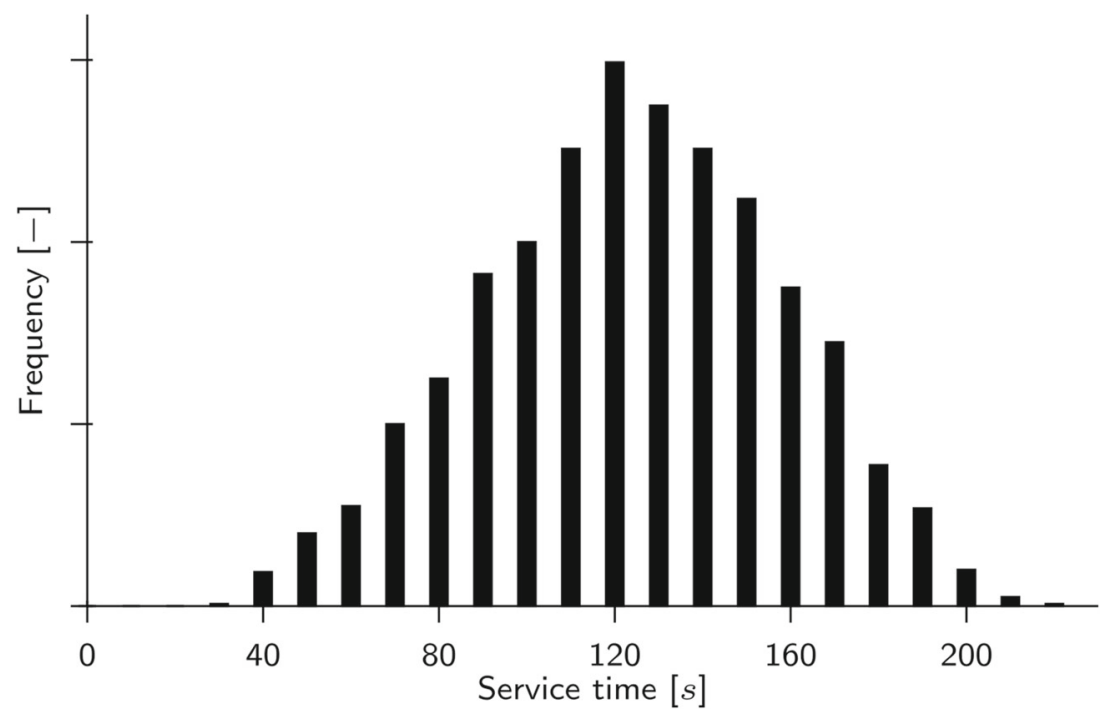


Table 4 Tested parameter configurations of the tier-captive single-aisle SBS/RS

\begin{tabular}{ll}
\hline Parameter & Value \\
\hline Number of tiers & $n_{\text {tier }} \in\{8,14,20,26,32,38,44,50\}$ \\
Number of slots per tier on each side of the aisle & $n_{\text {slot }}=\{100,200\}$ \\
Storage depth of the rack & $s d \in\{1,3,5\}$ \\
Number of buffers per tier on each side of the aisle & $n_{\text {buf }}=1$ \\
Filling degree of the storage rack & $f \in\{10 \%, 25 \%, 50 \%, 75 \%, 90 \%, 95 \%, 98 \%\}$ \\
Distance between two storage slots & $\Delta x=0,5 \mathrm{~m}$ \\
Distance between two tiers & $\Delta y=0,4 \mathrm{~m}$ \\
Vertical distance between the first tier and the I/O point & $l_{I / O}=0 \mathrm{~m}$ \\
Lift velocity & $v_{l i f t}=5 \frac{\mathrm{m}}{\mathrm{s}}$ \\
Lift acceleration/deceleration rate & $a_{\text {lift }}=7 \frac{\mathrm{m}}{\mathrm{s}^{2}}$ \\
Time to transfer a tote to and from the lift & $t_{t_{L}}=1.4 \mathrm{~s}$ \\
Shuttle velocity & $v_{\text {shuttle }}=2 \frac{\mathrm{m}}{\mathrm{s}}$ \\
Shuttle acceleration/deceleration rate & $a_{\text {shuttle }}=2 \frac{\mathrm{m}}{\mathrm{s}^{2}}$ \\
Time needed for transferring a tote to and from the shuttle out of the buffer & $t_{t_{b}}=8.4 \mathrm{~s}$ \\
Time needed for transferring a tote to and from the shuttle out of the rack & $t_{t_{n}} \in\{8.4 ; 12.7 ; 16.8 ; 21.3 ; 25.6 \mathrm{~s}\}$ \\
\hline
\end{tabular}

11. Shuttle travels to the storage position where the ordered tote is situated.

12. Tote is transferred from the rack to the shuttle.

13. Shuttle relocates the loaded tote if necessary or travels to the output-buffer.

14. Ordered tote is transferred from the shuttle to the output-buffer.

15. Tote is waiting for the lift.

16. Lift travels to the respective tier.

17. Tote is transferred from the output buffer to the lift.

18. Lift travels to the $\mathrm{I} / \mathrm{O}$ point.

19. Tote is transferred from the lift to the I/O point.

For each transaction, the lifts' travel times were calculated according to Eqs. 1-5 and the shuttles' travel times according to Eqs. 6-11, depending on the handling cycle strategy. The times needed for the transfer from and to the shuttle were calculated according to Eqs. 14 and 15, depending on the storage depth. The time needed for the relocation cycle was calculated with the Eqs. 16-21, depending on the storage depth and the filling degree of the rack. The waiting times for lifts and shuttles were determined by the open queuing model with limited capacity (22)-(30), which also calculates the throughput of one tier. With Eq. 24, the throughput of a single aisle can be calculated. The parameters used in this equation are shown in Table 4. These parameters are contributed by a European material handling provider. Figures 9 and 10 show the results of the analytical model and the simulation.

In Figs. 9 and 10, the curve can be seen, the DES results are right in between the two curves of the results from the analytical model. In the DES, the shuttles execute a combination of single command cycle and dual command cycle, so the curve of the DES should be between these two curves of the analytical calculation. These plots illustrate the proximity of the open queueing system approach to the real system. Figures 9 and 10 illustrate the throughput of multiple-deep racks (Fig. 9 of triple-deep racks with a filling degree of $90 \%$, Fig. 10 of fivefold-deep racks with a filling degree of $90 \%$ ). The reason why the simulation model is a mix of single and dual command cycle is that, for a fully dual command cycle, the input to the various tiers must be controlled so that the waiting times for the shuttle are kept to a minimum. To solve this, a complex control policy is required, and it is not the aim of this paper to provide such a control policy.

The comparison between analytical calculation and simulation concerning the throughput of shuttles with dual

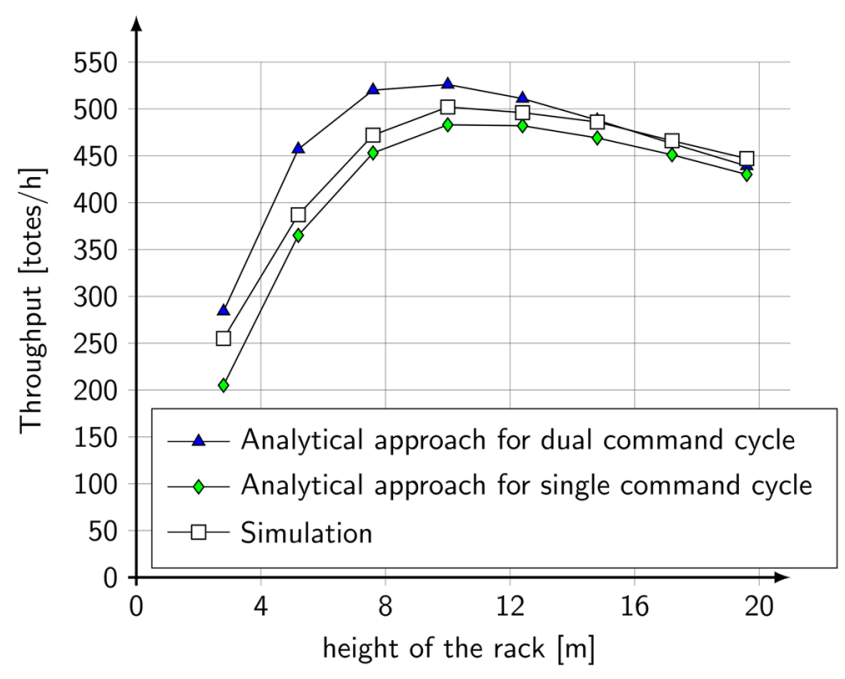

Fig. 9 Throughput of a rack with 100-m length and triple-deep storage 


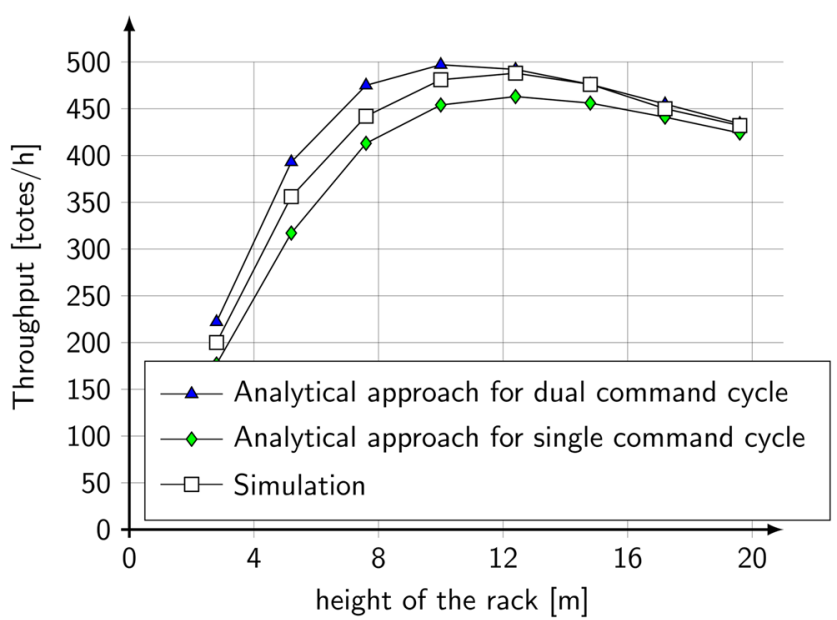

Fig. 10 Throughput of a rack with 100-m length and fivefold-deep storage

command cycle and different storage depths are shown in Figs. 11 and 12. The mean squared error between simulation and analytical approach between a filling degree of $10 \%$ and $98 \%$ is $0.98 \%$ for triple-deep storage (see Fig. 11) and $2.66 \%$ for fivefold-deep storage (see Fig. 12). Thus, accurate results are to be expected from this analytical approach. This comparison is performed to validate the approach of the cycle times of the shuttles for multi-deep storage.

The influence of the storage depth on the throughput of levels with different storage depths at a filling level of $90 \%$ is shown in Fig. 13. As can be seen, the throughput decreases with increasing storage depth. The influence on a single tier is relatively high. This effect loses significance when looking at a whole aisle, as shown in the following section. The reason for this is that the length of the aisle is only half in order to get the same amount of storage space when switching from single to double deep. In the following section, the influence of the storage depth on a whole aisle is shown in more detail and here the influence on the throughput is given, too.

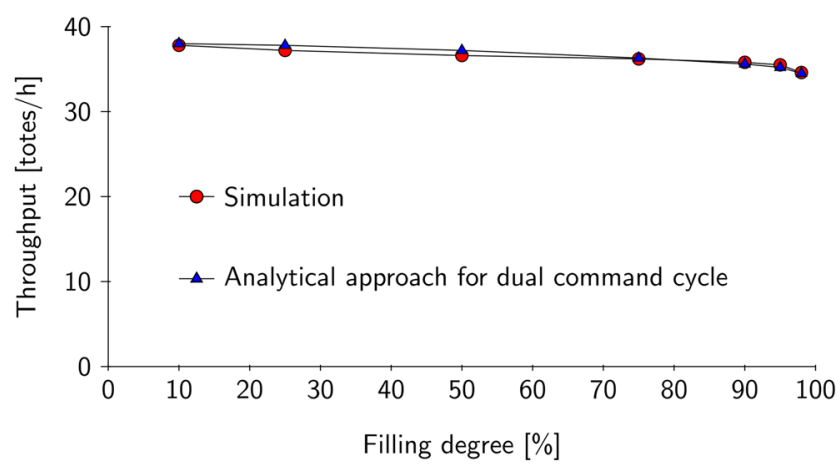

Fig. 11 Throughput of one tier with 100-m length and triple-deep storage

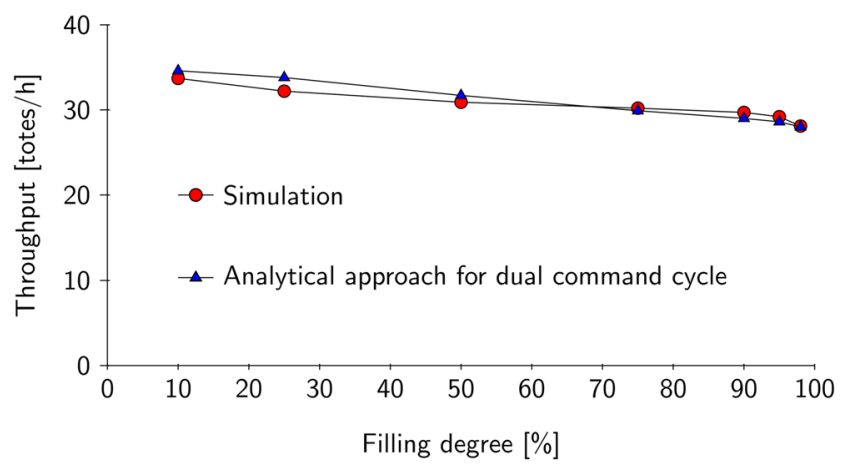

Fig. 12 Throughput of one tier with 100-m length and fivefold-deep storage

\subsection{Optimization example}

The optimization example is based on the requirements listed in Table 5.

The system requires a storage capacity of 25,000 spaces per aisle and there are no height and length restrictions. The maximum storage depth of the rack is five. The width of an aisle is given as $W=1 m+0.7 m \cdot s d$. For the footprint, the space required for buffers, lifts, and I/O points in front of the aisle are not considered. Due to [9, 11, 23], the throughput of multiple-aisle SBS/RS can be obtained by calculation the performance of one aisle.

The lift and shuttle parameters take the same values as in Section 5.1.

The procedure of this optimization is as follows:

- Determination of the length of an aisle by a given storage depth and for a different number of tiers.

- Determination of the throughput according to Fig. 3 with a filling degree of $90 \%$.

- Determination of the maximum throughput of the configurations determined in the first step.

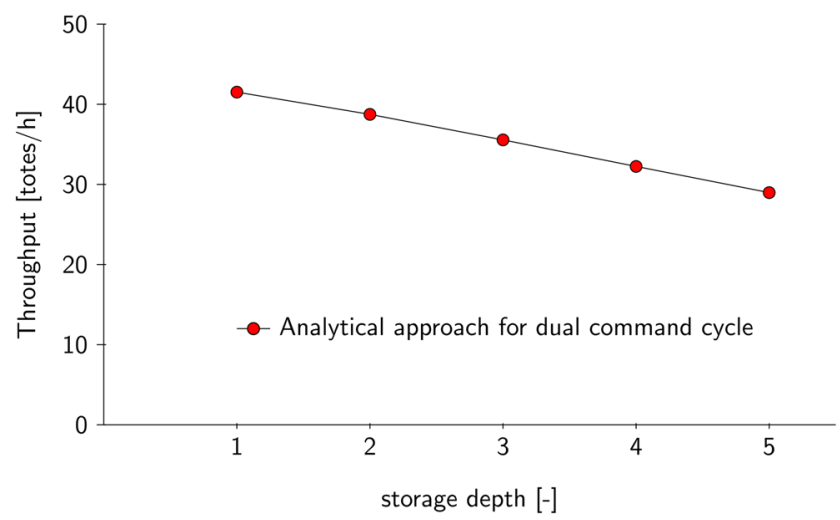

Fig. 13 Throughput of one tier with 100-m length with different storage depths and a filling degree of $90 \%$ 
Table 5 Requirements for the tier-captive SBS/RS

\begin{tabular}{ll}
\hline Parameter & Value \\
\hline Storage capacity & $N=25000$ \\
Number of aisles & $A \in\{1,2,3,4,5\}$ \\
Storage depth of the rack & $s d \in\{1,2,3,4,5\}$ \\
\hline
\end{tabular}

The results of these calculations are displayed in Table 6 . The ratio of $\frac{n_{\text {tier }}}{n_{\text {slot }}}$ is chosen for the highest throughput at each configuration. The results for a single aisle with different storage depths are visualized in Fig. 5.2. The minimum requirement of 25,000 storage locations leads to the fact that the length of the rack decreases an with increasing storage depth and with increasing number of aisles. The number of tiers for maximum throughput decreases with increasing number of aisles. The storage depth influences the number of tiers as well. While up until a storage depth of 3 , the number of tiers generally decreases, with a further increase of storage depth, it slightly increases, too. The throughput generally increases up to a storage depth of 3 and it slightly decreases at higher storage depths. The footprint of the SBS/RS as well as its throughput $\left(\vartheta_{\text {system }}\right)$ increase with the number of aisles.

Due to the fact that all configurations have approximately the same number of storage positions, the costs of the SBS/RS mainly depend on the annual costs of its footprint as well as the annualized costs of lifts and shuttles (see also [24]). To compare the different configurations of the example, the same cost structure as in [24] is assumed (i.e., 50e per $\mathrm{m}^{2}$ footprint and year, 10 years of service, $10 \%$ interest rate, investment costs of $10,000 e$ per shuttle, $50,000 e$ per lift and $30 e$ per storage position). In order to take into account the different storage depths, the costs per shuttle increase with storage depth by $1,000 e$. The configuration with one aisle, 26 tiers, 161 slots, and triple depth storage results in the lowest costs $(137,000 e)$ of all configurations. The visualization of a single aisle and different storage depths is shown in Fig. 14.

Table 6 Optimization example

\begin{tabular}{|c|c|c|c|c|c|c|c|c|}
\hline A & $n_{\text {tier }}$ & $n_{\text {slot }}$ & $s d$ & $\mathrm{~N}$ & Footprint $\left[m^{2}\right]$ & $\vartheta_{\text {aisle }}\left[\frac{1}{h}\right]$ & $\vartheta_{\text {system }}\left[\frac{1}{h}\right]$ & Costs $[e]$ \\
\hline 1 & 40 & 313 & 1 & 25040 & 375.6 & 448 & 448 & 144000 \\
\hline 1 & 31 & 202 & 2 & 25048 & 338.8 & 525 & 525 & 138000 \\
\hline 1 & 26 & 161 & 3 & 25116 & 418.6 & 543 & 543 & 137000 \\
\hline 1 & 30 & 105 & 4 & 25200 & 346.5 & 541 & 541 & 142000 \\
\hline 1 & 25 & 100 & 5 & 25000 & 400 & 544 & 544 & 140000 \\
\hline 2 & 36 & 174 & 1 & 25056 & 417.6 & 509 & 1018 & 152000 \\
\hline 2 & 22 & 143 & 2 & 25168 & 543.4 & 576 & 1152 & 147000 \\
\hline 2 & 21 & 100 & 3 & 25200 & 520 & 590 & 1180 & 147000 \\
\hline 2 & 21 & 75 & 4 & 25200 & 495 & 587 & 1174 & 148000 \\
\hline 2 & 22 & 57 & 5 & 25080 & 456 & 573 & 1146 & 149000 \\
\hline 3 & 27 & 155 & 1 & 25110 & 558 & 559 & 1677 & 160000 \\
\hline 3 & 20 & 105 & 2 & 25200 & 598.5 & 606 & 1818 & 158000 \\
\hline 3 & 19 & 74 & 3 & 25308 & 577.2 & 615 & 1845 & 158000 \\
\hline 3 & 19 & 55 & 4 & 25080 & 544.5 & 606 & 1818 & 157000 \\
\hline 3 & 20 & 42 & 5 & 25200 & 504 & 587 & 1761 & 159000 \\
\hline 4 & 21 & 149 & 1 & 25032 & 715.2 & 586 & 2344 & 172000 \\
\hline 4 & 18 & 87 & 2 & 25056 & 661.2 & 627 & 2508 & 168000 \\
\hline 4 & 18 & 58 & 3 & 25056 & 603.2 & 631 & 2524 & 167000 \\
\hline 4 & 18 & 44 & 4 & 25344 & 580.8 & 618 & 2472 & 168000 \\
\hline 4 & 19 & 33 & 5 & 25080 & 528 & 595 & 2380 & 168000 \\
\hline 5 & 20 & 125 & 1 & 25000 & 750 & 608 & 3040 & 183000 \\
\hline 5 & 16 & 79 & 2 & 25280 & 750.5 & 642 & 3210 & 181000 \\
\hline 5 & 16 & 53 & 3 & 25440 & 689 & 643 & 3215 & 180000 \\
\hline 5 & 17 & 37 & 4 & 25160 & 610.5 & 626 & 3130 & 178000 \\
\hline 5 & 20 & 25 & 5 & 25000 & 500 & 599 & 2995 & 178000 \\
\hline
\end{tabular}


Fig. 14 Optimization example

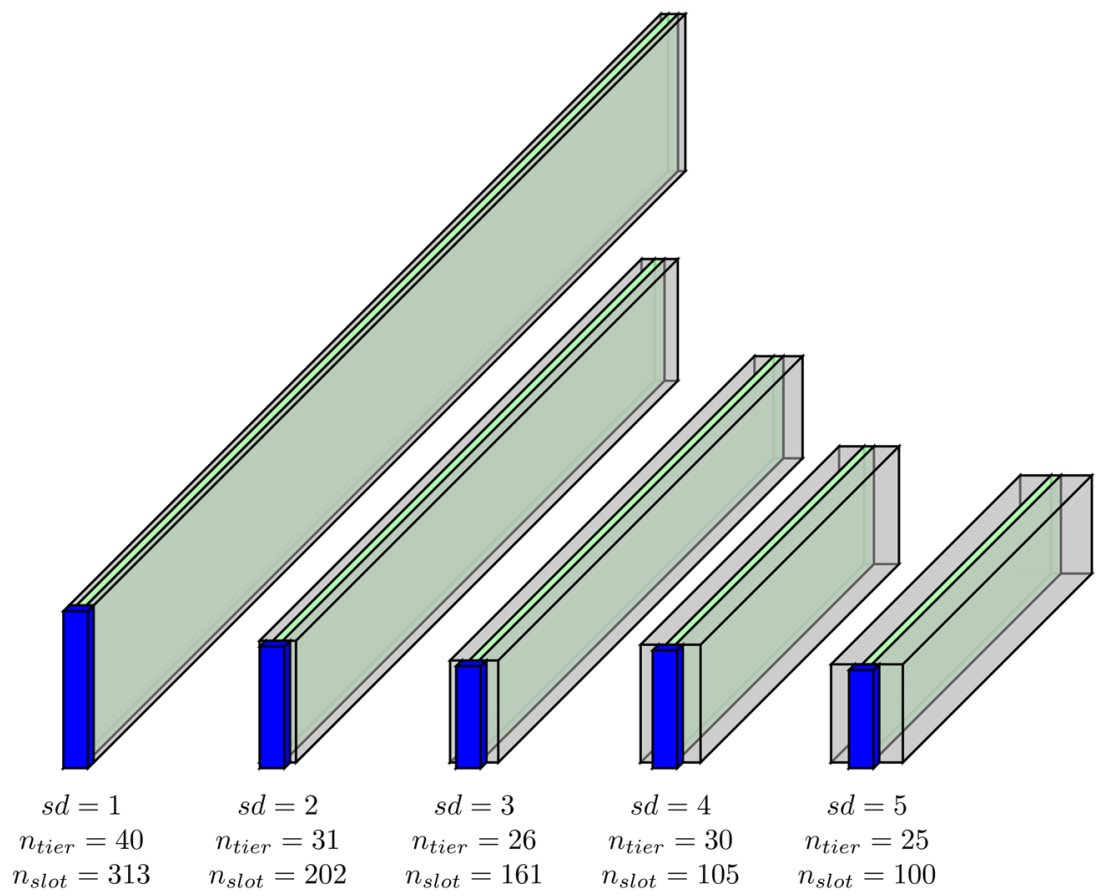

\section{Conclusion}

Tier-captive SBS/RS are increasingly used in many industries due to their high system performance. However, few analytical decision-making tools are available to evaluate the performance of an SBS/RS. The existing methods discuss SBS/RS with single- or double-deep storage. One uses an open queueing system with limited capacity to estimate the interactions between lifts and shuttles. The second uses open queueing networks to discuss the interactions between shuttles and lifts. This open queueing network approach has the limitation, that it only calculates the waiting times between lifts and shuttles within one transaction and it is not possible to deduce the throughput of a whole aisle. The relevance of this multipledeep SBS/RS compared with a single lane stacker is that, although the SBS/RS stores multiple-deep, the throughput is significantly higher than in a single lane stacker. The throughput of a single lane stacker can be estimated as the throughput of a single tier within the SBS/RS, as shown in the previous chapter. This estimation is true for a single lane stacker where the length of the rack is the limitation factor for the throughput. As can be seen through this comparison, the throughput of the SBS/RS is about ten times higher than that of a single-lane stacker with the same specifications.

As a result, a method for calculating the performance of tier-captive single-aisle SBS/RS is presented which is easy to use and equally delivers accurate results. The system is modelled as a continuous-time open queueing system with limited capacity. Subsequently, the interarrival and service times are evaluated by a cycle time model of lifts and shuttles with discrete spatial values. The feature of the presented cycle time model is that it can also treat multipledeep storage by using an approach with probabilities of the relocation process and the estimated time needed for this process. The accuracy of the analytical model in comparison to a DES is validated by a numerical study in which the four mainly different configuration parameters, length, height, storage depth, and filling degree, are discussed.

In general, the presented approach reaches a high approximation quality. The storage process is modelled by an open queueing model with limited capacity, which is the best approximation of reality. In addition, the real distributions of the interarrival times and service times were used in this analytical model. The accuracy is proven by comparing the results of the analytical approach to a discrete event simulation of a real shuttle-based storage and retrieval system. In addition to verifying the final results of the system, the results within the system, for example, the cycle times of the shuttles within a shift, were analyzed to determine the quality of the approach.

Finally, it is outlined how the presented queueing model can be used to design tier-captive SBS/RS for given requirements, such as storage capacity, throughput, height, and length. The resulting costs of the designs are also calculated. The example shows the influence of the storage depth and the number of aisles. This approach can be used to design a storage system for any predefined requirements, which is an advantage for any provider of SBS/RS. For example, when designing a new storage system, this approach can be used to calculate the key data for building such a system in a simple and accurate manner. 
The presented assumptions are similar to the SBS/RS of a European material handling provider.

Further work will be dedicated to the SBS/RS with alternative system configurations and different storage management polices. Such a system may have a varying number of tiers served by a shuttle. Another interesting aspect is the storage of different tote sizes within the same tier, resulting in a variety of storage depths. A storage policy used in existing systems is to design the storage process so that the totes are sorted to batches and no relocation cycle is required. All these points will be incorporated into further work.

Acknowledgments Open access funding provided by TU Wien (TUW).

Funding information This work was financially supported by the TU Wien University Library, through its Open Access Funding Programme.

Open Access This article is licensed under a Creative Commons Attribution 4.0 International License, which permits use, sharing, adaptation, distribution and reproduction in any medium or format, as long as you give appropriate credit to the original author(s) and the source, provide a link to the Creative Commons licence, and indicate if changes were made. The images or other third party material in this article are included in the article's Creative Commons licence, unless indicated otherwise in a credit line to the material. If material is not included in the article's Creative Commons licence and your intended use is not permitted by statutory regulation or exceeds the permitted use, you will need to obtain permission directly from the copyright holder. To view a copy of this licence, visit http://creativecommons. org/licenses/by/4.0/.

\section{References}

1. Borovinšek M, Ekren B, Burinskienė A, Lerher T (2017) Multiobjective optimisation model of shuttle-based storage and retrieval system. Transport 32(2):120-137

2. Eder M (2019) An analytical approach for a performance calculation of shuttle-based storage and retrieval systems. Prod Manuf Res 7(1):255-270. https://doi.org/10.1080/21693277.2019.1619102

3. Eder M, Kartnig G (2016) Durchsatzoptimierung von shuttlesystemen mithilfe eines analytischen berechnungsmodells. Logistics Journal: Proceedings 10:113-126

4. Eder M, Kartnig G (2016) Throughput analysis of s/r shuttle systems and ideal geometry for high performance. FME Trans 44(2):174-179

5. Eder M, Kartnig G (2018) Calculation method to determine the throughput and the energy consumption of $\mathrm{s} / \mathrm{r}$ shuttle systems. FME Trans 46(3):424-428

6. Ekren B, Sari Z, Lerher T (2015) Warehouse design under classbased storage policy of shuttle-based storage and retrieval system. IFAC-PapersOnLine 48(3):1152-1154

7. Ekren B, Heragu SS (2012) Performance comparison of two material handling systems: Avs/rs and cbas/rs. Int J Prod Res 50(15):4061-4074

8. Ekren BYea (2017) A queuing network approach for performance estimation of shuttle based storage and retrieval system design. In: XXII International conference on material handling constructions and logistics - MHCL 2017
9. Epp M, Wiedemann S, Furmans K (2017) A discrete-time queueing network approach to performance evaluation of autonomous vehicle storage and retrieval systems. Int J Prod Res 55(4):960-978

10. Ha Y, Chae J (2018) Free balancing for a shuttle-based storage and retrieval system. Simul Model Pract Theory 82:12-31

11. Heragu SS, Cai X, Krishnamurthy A, Malmborg CJ (2011) Analytical models for analysis of automated warehouse material handling systems. Int J Prod Res 49(22):6833-6861

12. Karnig G, Oser J (2014) Throughput analysis of s/r shuttle systems. In: International material handling research colloquium 2014

13. Kriehn T, Schloz F, Wehking KH, Fittinghoff M (2018) Impact of class-based storage, sequencing of retrieval requests and warehouse reorganisation on throughput of shuttle-based storage and retrieval systems. FME Trans 46(3):320-329

14. Lerher $\mathrm{T}$ (2013) Modern automation in warehousing by using the shuttle based technology. Automation Systems of the 21st Century: New Technologies, Applications and Impacts on the Environment \& Industrial Processes, pp 51-86

15. Lerher T (2016) Travel time model for double-deep shuttle-based storage and retrieval systems. Int J Prod Res 54(9):2519-2540

16. Lerher $T$ (2017) Design of experiments for identifying the throughput performance of shuttle-based storage and retrieval systems. Procedia Eng 187:324-334

17. Lerher T, Akpunar xA et al (2016) Simulation-based energy and cycle time analysis of shuttle-based storage and retrieval system. 14th International Material Handling Research Coloquium (IMHRC 2016)

18. Lerher T, Ekren B, Dukic G, Rosi B (2015) Travel time model for shuttle-based storage and retrieval systems. Int J Adv Manuf Technol 78(9-12):1705-1725

19. Lerher T, Ekren B, Sari Z, Rosi B (2016) Method for evaluating the throughput performance of shuttle based storage and retrieval systems. Tehnicki vjesnik/Technical Gazette 23(3):715-723

20. Lerher T, Ekren Y, Sari Z, Rosi B (2015) Simulation analysis of shuttle based storage and retrieval systems. International Journal of Simulation Modelling (IJSIMM) 14(1):1-13

21. Lerher T, Rosi B, Ekren Y, Sari Z (2012) A model for throughput performance calculations of shuttle based storage and retrieval systems. Techn vjesnik 19(4):709-715

22. Lerher T, Zrnić N, Jerman B (2016) Throughput and energy related performance calculations for shuttle based storage and retrieval systems. Nova Science Publishers, Incorporated

23. Marchet G, Melacini M, Perotti S, Tappia E (2012) Analytical model to estimate performances of autonomous vehicle storage and retrieval systems for product totes. Int J Prod Res 50(24): 7134-7148

24. Marchet G, Melacini M, Perotti S, Tappia E (2013) Development of a framework for the design of autonomous vehicle storage and retrieval systems. Int J Prod Res 51(14):4365-4387

25. Sari Z, Ghomri L, Ekren B, Lerher T (2014) Experimental validation of travel time models for shuttle-based automated storage and retrieval system. In: International material handling research colloquium, pp 23-27

26. Smith JM (2004) Optimal design and performance modelling of M/G/1/K queueing systems. Math Comput Model 39(9-10):10491081

27. Trummer W, Jodin D (2014) Welche Leistung haben ShuttleSysteme? In: HEbezeuge Fördermitttel

28. VDI 3561 (1973) Testspiele zum Leistungsvergleich und zur Abnahme von Regalförderzeugen

29. Wang Y, Mou S, Wu Y (2016) Storage assignment optimization in a multi-tier shuttle warehousing system. Chin J Mech Eng 29(2):421-429

Publisher's note Springer Nature remains neutral with regard to jurisdictional claims in published maps and institutional affiliations. 